\title{
Raúl Prebisch \\ y la agenda del desarrollo en los albores del siglo XXI
}

José Antonio Ocampo*

El centenario del nacimiento de Raúl Prebisch constituye una invaluable oportunidad para retomar las ideas de este gran latinoamericano, uno de los pensadores del mundo en desarrollo que más influencia ha tenido en los debates económicos mundiales. Sus ideas han sido objeto de severas críticas, muchas de ellas referidas, sin embargo, a caricaturas de su pensamiento o a las distorsiones que éste experimentó en la práctica, más que a su obra intelectual. Sacar sus formulaciones de su contexto histórico ha sido igualmente una constante, aun por algunos de sus seguidores. Debe recordarse, en particular, que muchas de ellas surgieron del colapso del sistema comercial y financiero internacional de la década de 1930, cuya reconstrucción apenas se iniciaba en los momentos en que publicó sus trabajos más influyentes (Prebisch, 1949, 1951 y 1952).

Este ensayo intenta mostrar la vigencia de algunas de las ideas esenciales en el pensamiento de Prebisch, y las reformula en función de los planteamientos más recientes de la CEPAL y de las contribuciones de otras escuelas económicas.

\footnotetext{
* Secretario Ejecutivo de la CEPAL (jaocampo@eclac.cl). El presente trabajo fue presentado en el seminario "La teoría del desarrollo en los albores del siglo XXI", organizado por la CEPAL para conmemorar el centenario del nacimiento de Raúl Prebisch. El autor agradece a Oscar Altimir, María Elisa Bernal, María Angela Parra y Rosemary Thorp los comentarios a una versión previa.
}

\section{Tres ejes centrales del pensamiento de Prebisch}

"La propagación universal del progreso técnico desde los países originarios al resto del mundo ha sido relativamente lenta e irregular" (Prebisch, 1951, p. 1). Esta afirmación, con la que se inicia la que es quizás su obra más conocida, tiene dos implicaciones metodológicas fundamentales. La primera es el reconocimiento del papel clave que tiene el progreso técnico en el crecimiento económico y, por ende, la importancia que revisten para los países en desarrollo los canales por los cuales dicho progreso se transmite desde los "países originarios": la demanda derivada de materias primas; la transferencia de tecnología como tal, incluida aquella incorporada en equipos productivos; el traslado a países en desarrollo de ramas productivas "maduras" en los países industrializados, y la participación de los países en desarrollo en sectores de dinamismo tecnológico. La segunda es la incapacidad de analizar la dinámica de los países en desarrollo con independencia de su posición dentro de la economía mundial. Sus procesos de desarrollo son cualitativamente diferentes a los de las naciones más avanzadas. Esto implica que no hay "etapas de desarrollo" uniformes, que el "desarrollo tardío" —el "capitalismo periférico", para emplear su propia terminología - tiene una dinámica diferente a la de las naciones que experimentaron un desarrollo más 
temprano y se transformaron en el "centro" de la economía mundial. ${ }^{1}$

Esta visión subyace la idea de un sistema económico mundial inherentemente jerarquizado, "centroperiferia", o "Norte-Sur", si se prefiere la terminología que se popularizó en los debates de la década de 1970. La esencia de esta visión es el énfasis en las asimetrías básicas que caracterizan a la economía mundial, y su persistencia a lo largo del tiempo, que contrastan con la concepción de la economía mundial como un contexto de relación entre pares, como un "campo de juego nivelado", para utilizar un concepto que se ha popularizado en los últimos años. En términos de las controversias recientes sobre crecimiento económico, esta visión alternativa de la economía mundial implica que las asimetrías que la caracterizan tienden a generar "divergencia" en los niveles de desarrollo, o al menos constituye un fuerte obstáculo a la "convergencia" que suponen las teorías ortodoxas de crecimiento económico. ${ }^{2}$

Estas asimetrías se reflejan, en primer lugar, en las estructuras productivas. De acuerdo con una formulación muy cercana al pensamiento de Prebisch, "en contraste con la estructura productiva de la periferia, especializada y heterogénea, la de los centros se caracteriza por ser diversificada y homogénea" (Rodríguez, 2001, p. 105). Dado que el cambio técnico se origina en los países del centro y ellos ostentan, además, una mayor capacidad de consumo, tienden a concentrar en cada momento las ramas de producción más dinámicas a nivel mundial. Esto genera, en la visión de Prebisch, una tendencia a la especialización de los países industrializados en productos de alta elasticidad-ingreso y de los periféricos en aquellos de baja elasticidad-ingreso (materias primas y, crecientemente, manufacturas en sus etapas maduras), que se refleja, a su vez, en una tendencia a la divergencia en los ritmos de crecimiento y/o a la aparición de problemas de balanza de pagos en los segundos, es decir, a una "brecha" o "estrangulamiento" externo. Estos problemas son particularmente severos durante los períodos de crisis, reflejando la alta vulnerabilidad cíclica de los

\footnotetext{
${ }^{1}$ La visión más acabada de estos puntos de vistas se encuentra, sin duda, en Furtado (1961)

${ }^{2}$ En esta formulación hacemos caso omiso de la controversia sobre los términos de intercambio, que ha recibido excesiva atención en los análisis de la obra de Prebisch. La atención se concentra, por lo tanto, en la divergencia de los niveles de desarrollo o, en términos de la controversia mencionada, en el papel que desempeña el deterioro de los términos de intercambio factoriales. Véase, sobre este tema, Ocampo (1991).
}

países en desarrollo frente a las perturbaciones provenientes del centro de la economía mundial.

En la visión de Prebisch, la superación de las asimetrías básicas del sistema internacional exige no sólo un cambio en la estructura económica internacional, sino también un esfuerzo por transformar las estructuras de los países periféricos, un "desarrollo desde dentro", para utilizar la terminología original de Prebisch, rescatada por Sunkel (comp., 1991). En términos de los debates más recientes, este "desarrollo desde dentro" es esencial porque la acumulación de capital humano y de capacidades tecnológicas propias ("capital conocimiento") y el desarrollo institucional son procesos esencialmente endógenos. De ahí la importancia decisiva de "programar el desarrollo", término empleado en las primeras etapas de la CEPAL y, en general, de diseñar estrategias estatales explícitas dirigidas a transformar las estructuras internas, para romper los obstáculos al desarrollo y permitir nuevas formas de integración a la economía mundial.

La industrialización fue vista inicialmente como la principal vía de transformación de la estructura productiva - de "difusión del progreso técnico"- y la sustitución de importaciones como su principal instrumento. Esta visión correspondía a las características del momento histórico en el cual estas concepciones fueron formuladas: la ausencia de un mercado dinámico de manufacturas a nivel internacional; ${ }^{3}$ la forma "empírica" como había surgido la estrategia de sustitución de importaciones, como respuesta al colapso de la economía internacional en los años treinta; y el pasado proteccionista que muchos países latinoamericanos habían compartido con Estados Unidos y varios países de Europa continental, aun durante la etapa de desarrollo primario-exportador. ${ }^{4}$ Las posibles ineficiencias de la sustitución de importaciones, particularmente en mercados altamente fragmentados, así como la necesidad de evitar que la industrialización se hiciese a costa de la agricultura o del desarrollo exportador, fueron evidentes para Prebisch desde sus primeros escritos en la CEPAL (Prebisch, 1949, secciones I y VI). Por este motivo, desde finales de los años cincuenta,

\footnotetext{
3 "No se hizo en esta etapa ningún hincapié en las exportaciones de manufacturas a los centros, dadas las condiciones desfavorables prevalecientes en ellos y la ausencia de una infraestructura industrial adecuada para tal efecto" (Prebisch, 1987, p. 17).

4 Sobre el proteccionismo latinoamericano durante la etapa exportadora, véase Cárdenas, Ocampo y Thorp (eds., 2000a, cap. 1). Sobre la comparación de los aranceles latinoamericanos con los de varios países de Europa continental y los Estados Unidos, véase Maddison (1989, pp. 45-47).
} 
Prebisch y la CEPAL pasaron a defender un "modelo mixto" que combinaba la sustitución de importaciones con la promoción de nuevas exportaciones, especialmente de origen industrial. ${ }^{6}$ A lo largo del tiempo, la visión de la CEPAL se volvió crecientemente proexportadora, aunque enemiga de un desmantelamiento abrupto de las estructuras de protección. ${ }^{7}$

La industrialización en los países periféricos implicaba, para Prebisch, un problema adicional: la industria moderna tenía una menor capacidad de absorber la mano de obra liberada por el sector rural. Este hecho se veía agravado por la dependencia tecnológica, que implicaba que la "mejor práctica" correspondía a patrones de uso de mano de obra inducidos por los niveles de desarrollo de los países industrializados. Por una y otra vía, los patrones de desarrollo se caracterizaban por una mayor "heterogeneidad estructural" de los sectores productivos, para emplear el término desarrollado posteriormente por Pinto (1970): mientras algunos trabajadores eran absorbidos en los sectores de alta productividad, una proporción, generalmente mayoritaria, quedaban relegados a sectores de baja productividad. Esta alta heterogeneidad interna era una de las fuerzas básicas que presionaba en forma adversa la distribución del ingreso, en países que, por lo demás, en el caso de América Latina habían heredado de etapas anteriores de su desarrollo una alta desigualdad y una elevada segmentación social.

Dentro de la estrategia mixta de desarrollo, los procesos de integración fueron vistos, desde comienzos de los años cincuenta, como elementos claves para racionalizar los costos de la sustitución de importaciones, tanto aquellos asociados a la ausencia de compe-

\footnotetext{
${ }^{5}$ Este es el término que utiliza un reciente estudio de historia económica (Cárdenas, Ocampo y Thorp, eds., 2000b, cap. 1).

${ }^{6}$ Esto coincide con lo que Prebisch identifica como la tercera etapa de su pensamiento (Prebisch, 1987, pp. 9-21). Una de sus expresiones más claras es, sin duda, Prebisch (1963).

7 Véanse, al respecto, tres versiones recientes de la historia del pensamiento de la CEPAL: Bielschowsky (1998), CEPAL (1998) y Rosenthal (2001). Las razones de esta visión (que tiene ahora, fundamentalmente, un interés histórico) estuvieron asociadas a tres consideraciones: i) los costos de transmisión que generaría el desmantelamiento de las estructuras de protección; ii) la idea de que era posible racionalizar la estructura de incentivos a las exportaciones para compensar los costos de la protección; y iii) que existía alguna complementariedad entre protección y desarrollo exportador; en particular, en presencia de economías de escala, el mercado local servía de "base" para la conquista de los mercados externos (un argumento que posteriormente Krugman, 1990, cap. 12, denominó "sustitución de importaciones como promoción de exportaciones"). Prebisch y la CEPAL siempre reconocieron, sin embargo, que los altos niveles de protección podían generar ineficiencias y sesgos antiexportadores insalvables.
}

tencia como a la ineficiencia que se derivaba de la escala subóptima de las plantas industriales. Una década más adelante, cuando las posibilidades de exportar manufacturas hacia los países industrializados comenzó a ser evidente, se les consideró igualmente un instrumento para acumular experiencia exportadora como antesala a la conquista de otros mercados. La integración regional - los diversos procesos subregionales, la ALALC/ALADI y el mercado común latinoamericano, en la propuesta más ambiciosa - se convirtió, de esta manera, en un elemento decisivo del "modelo mixto" impulsado por Prebisch y la CEPAL desde fines de los años cincuenta (CEPAL, 1959). Era vista, así, como la forma para ampliar los espacios dentro de los cuales tenía lugar el "desarrollo desde dentro", para tornarlo más eficiente.

Las asimetrías internacionales características del sistema "centro-periferia", la necesidad de adoptar estrategias activas de "desarrollo desde dentro", incluidas aquellas dirigidas a enfrentar los problemas especiales que genera la "heterogeneidad estructural" y el papel crítico de la integración regional, constituyen tres ejes centrales del pensamiento de Prebisch. El resto de este artículo muestra la relevancia que tienen en la agenda del desarrollo de comienzos del siglo XXI.

\section{Las asimetrías internacionales}

La tendencia a la ampliación de las desigualdades ha sido una característica persistente de la economía mundial durante los dos últimos siglos. Los estudios empíricos indican, en efecto, que la convergencia en los niveles de ingreso por habitante ha sido un evento más bien escaso y se ha limitado, de hecho, a los países más industrializados después de la Segunda Guerra Mundial y, más específicamente, durante la "edad de oro" (1950-1973). No fue una característica de los países industrializados antes de esa guerra (Maddison, 1991), ni tampoco de los países en desarrollo desde entonces (Ros, 2000, cap. 1). Sin embargo, ha habido episodios de rápido crecimiento en el mundo en desarrollo en algunos períodos: América Latina fue, de hecho, la región de mayor crecimiento en el mundo entre las dos guerras mundiales y algunos países asiáticos lo han sido en algunos períodos posteriores a la segunda de ellas: los países petroleros del Medio Oriente, los tigres asiáticos, y China y la India, en diferentes subperíodos (Maddison, 1995). Sin embargo, salvo en el Japón, estos procesos no han logrado una convergencia con los patrones de desarrollo del mundo industrializado, y en la mayoría de los casos se han 
interrumpido. Cabe agregar que, aun al interior de América Latina, no hay ninguna tendencia clara a la convergencia: las desigualdades que se establecieron hace cerca de un siglo han tendido a mantenerse a lo largo del tiempo (Cárdenas, Ocampo y Thorp, eds., 2000a, cap. 1, y 2000b, cap. 1).

La persistencia e incluso la ampliación de estas desigualdades internacionales ha tenido lugar en medio del impresionante proceso de industrialización que han experimentado los países en desarrollo en el último medio siglo (y América Latina desde antes). Este proceso se ha traducido, por lo tanto, en una mayor diversificación de la estructura productiva del mundo en desarrollo, excepto en las regiones más atrasadas. No obstante, han subsistido importantes asimetrías en la estructura productiva a nivel mundial: la alta concentración del progreso técnico en los países del centro, que determina sus fuentes de competitividad; su continuo predominio en la producción de maquinaria y equipo e igualmente su papel dominante en la conformación de las grandes empresas trasnacionales.

La consecuencia más importante de las asimetrías de la economía mundial es que las oportunidades económicas de los países en desarrollo siguen estando determinadas en gran medida por su posición dentro de esta jerarquía internacional. Hay ciertamente "propagación de progreso técnico" desde el centro, a través de los canales ya mencionados. No obstante, utilizando los términos de Prebisch, dicha propagación ha seguido siendo "relativamente lenta e irregular", y sus frutos se han distribuido desigualmente en los países en desarrollo, manteniendo o incluso ampliando su "heterogeneidad estructural". Dentro del "objetivo móvil" que representa la frontera tecnológica mundial (Pérez, 2001), pocos países - y pocos sectores y empresas dentro de ellos - logran moverse más rápido y reducir así su atraso tecnológico; muchos otros sólo logran avanzar al ritmo de la frontera y no pocos se quedan rezagados. ${ }^{8}$

Por otra parte, la alta vulnerabilidad de los países en desarrollo frente a perturbaciones externas continúa siendo notoria e incluso ha tendido a incrementarse con la creciente integración de la economía internacional. Sin embargo, la naturaleza de la vulnerabilidad ha ido cambiando en el último medio siglo. Aunque la transmisión de los choques externos a través del comercio - tanto en forma directa, a través de menores ventas,

\footnotetext{
${ }^{8}$ Véase en Katz (2000) y CEPAL (2001a) información reciente sobre este tema para el caso latinoamericano.
}

como indirectamente, a través del deterioro cíclico de los términos de intercambio - ha continuando siendo importante, los choques financieros han pasado a ocupar un papel protagónico, reviviendo, por lo demás, patrones que ya se habían observado en el pasado en muchos países latinoamericanos, especialmente durante el auge y colapso financiero de las décadas de 1920 y 1930.

La vulnerabilidad es el resultado de asimetrías básicas en las estructuras financieras y en el funcionamiento macroeconómico, particularmente en la profundidad del desarrollo financiero y en el grado de autonomía macroeconómica de los países (Ocampo, 2001c). Las asimetrías financieras son fundamentalmente cuatro: i) la disparidad entre el tamaño de los mercados financieros de los países en desarrollo y las presiones especulativas que enfrentan; ${ }^{9}$ ii) la naturaleza de las monedas en las cuales está denominada la deuda externa; iii) las estructuras de plazos que proporcionan los mercados financieros, y iv) el alcance de los mercados secundarios. Estas tres últimas características implican que, en los países en desarrollo, aquellos agentes que pueden acceder a los mercados internacionales (el gobierno y las grandes empresas) enfrentan descalces de monedas, en tanto que aquellos que carecen de dicho acceso (las empresas pequeñas) enfrentan descalces de plazos, pero es en general imposible disponer de una estructura financiera que evite simultáneamente ambos riesgos. En conjunto, esto quiere decir que los mercados financieros de los países en desarrollo son significativamente más "incompletos" que los internacionales y, por ende, que una parte de la intermediación financiera debe llevarse a cabo necesariamente a través del mercado internacional. Significa, también, que la integración financiera internacional es una integración entre socios desiguales. ${ }^{10}$

Por su parte, las asimetrías macroeconómicas están asociadas al hecho de que las monedas internacionales son las monedas de los países industrializados y a la naturaleza de los flujos de capital: mientras los flujos de capital entre países desarrollados tienen un carácter anticíclico (Eatwell y Taylor, 2000), los flujos entre países desarrollados y países en desarrollo tienen un carácter claramente procíclico. Este comportamiento está asociado a la índole residual de los flujos de capital hacia los países en desarrollo o, para utilizar los términos de Palma (2001), a la naturaleza

\footnotetext{
${ }^{9}$ Véase, por ejemplo, Council on Foreign Relations (1999), cap. III. ${ }^{10}$ CEPAL (2000), cap. 8, y Studart (1996).
} 
de "deudores de última instancia" que tienen dichos países.

El resultado conjunto de estos factores es que mientras los países industrializados tienen mayores márgenes para adoptar políticas macroeconómicas anticíclicas - margen que es ciertamente mayor en los Estados Unidos que en el resto de las economías industrializadas, gracias a que dispone de la principal moneda internacional-, lo que induce una respuesta estabilizadora de los mercados financieros, las economías de los países en desarrollo carecen en gran medida de dichos márgenes, ya que los mercados financieros tienden a agudizar el ciclo y los actores del mercado esperan que las autoridades se comporten en forma procíclica. En una perspectiva histórica, esto indica que, mientras los países industrializados lograron liberarse en gran medida de las reglas del juego del patrón oro, dichas reglas continúan determinando el comportamiento macroeconómico de los países en desarrollo, e incluso se han vuelto cada vez más relevantes. De esta manera, las asimetrías macroeconómicas entre centro y periferia características de la era del patrón oro (Triffin, 1968; Aceña y Reis, eds., 2000) se han agudizado. Los arreglos multilaterales, especialmente la intervención del Fondo Monetario Internacional, aunque proporcionan alivios coyunturales, están dirigidos a garantizar la adopción de paquetes de austeridad durante las crisis — de una "(macro)economía de depresión", para usar la expresión de Krugman (1999) — En forma más estricta, lo que caracteriza a las economías de los países en desarrollo es la tendencia a alternar fases de "macroeconomía de bonanza" con fases de "macroeconomía de depresión" (CEPAL, 2000, cap. 8, y 2001b).

A las consideraciones anteriores cabe agregar que existe una asimetría adicional en la economía internacional, asociada con el contraste entre la creciente movilidad de capitales y la restricción a la movilidad internacional de mano de obra, especialmente de aquella con menores grados de calificación. Como lo ha señalado Rodrik (1997), las asimetrías en la movilidad internacional de distintos factores de producción generan sesgos en la distribución del ingreso en favor de los factores más móviles y en contra de los menos móviles. Estas asimetrías tienen un componente "centro-periferia", en la medida en que los países en desarrollo tienen una abundancia relativa de los factores de producción menos móviles: mano de obra con bajos niveles de calificación y recursos naturales. De hecho, la ausencia de movilidad internacional de mano de obra fue vista por Prebisch (1951) como una de las imper- fecciones básicas de la economía internacional, que desempeñaba un papel esencial en la tendencia al deterioro de los términos de intercambio de los países en desarrollo.

Por último, es necesario agregar que la economía internacional se caracteriza también por imperfecciones básicas que tienen un carácter "sistémico" más que de "centro-periferia". La primera es el contraste entre el desarrollo dinámico de los mercados y el rezago en la construcción de una gobernabilidad global, que ha conducido a un suministro subóptimo de "bienes públicos globales" (Kaul, Grunberg y Stern, comps., 1999). La segunda es la enorme diferencia entre la rápida globalización de algunos mercados y la notoria ausencia de una verdadera agenda social internacional o, con más precisión, la carencia de instrumentos efectivos de carácter internacional para garantizar el cumplimiento de las metas de desarrollo que se reiteran de manera periódica, más recientemente en la Declaración del Milenio de las Naciones Unidas. La tercera es el carácter incompleto de la agenda internacional, que tiene también en alguna medida dimensiones "centroperiferia", dada la ausencia de temas de alto interés para los países en desarrollo, como la movilidad internacional de mano de obra o la liberalización acelerada, por los países desarrollados, de mercados de alto interés para los países en desarrollo (Ocampo, 2001a).

Este análisis indica que la agenda global debe incluir tanto los temas "sistémicos", asociados a la provisión de "bienes públicos globales", como aquellos relacionados con la corrección de las asimetrías internacionales existentes. Estos temas "centro-periferia" incluyen fundamentalmente la corrección de las asimetrías en materia productiva y tecnológica, de las asimetrías financieras y macroeconómicas que inducen las elevadas vulnerabilidades cíclicas de los países en desarrollo, y de aquellas asociadas a los distintos grados de movilidad internacional de los factores de producción.

A partir de la creación de la UNCTAD, bajo influencia directa de Prebisch, ${ }^{11}$ se reconoció explícitamente la necesidad de corregir las asimetrías que caracterizaban, y siguen caracterizando, al sistema económico internacional. Los compromisos en materia de flujos de asistencia oficial para el desarrollo y el "trato especial y diferenciado" a los países en desarrollo en materia comercial fueron algunos de los resultados

11 Véase, por ejemplo, su primer informe a la UnCTAD (Prebisch, 1964). 
parciales, aunque relativamente frustrantes, de este esfuerzo por construir un "nuevo orden económico internacional". Esta visión se ha erosionado radicalmente en las últimas décadas (CEPAL, 2001c, cap.VIII) y ha sido sustituida por un paradigma alternativo, según el cual el objetivo básico en el reordenamiento de la economía internacional debe ser el de asegurar un "campo de juego nivelado" que garantice el funcionamiento eficiente de las libres fuerzas del mercado. En este esquema, las ganancias fundamentales para los países en desarrollo residen en el eventual desmonte del proteccionismo de los países industrializados a los sectores "sensibles", las garantías que ofrece al desarrollo exportador un marco comercial internacional con reglas claras y estables, y el diseño de políticas macroeconómicas preventivas que sirvan para "autoprotegerse" contra la volatilidad financiera internacional. La corrección de las asimetrías internacionales sólo se mantiene, en esta visión, en el reconocimiento de la responsabilidad internacional hacia los países menos adelantados, replicando a nivel internacional la visión de la política social como una estrategia de focalización de las acciones del Estado hacia los sectores más pobres.

Si bien todas estas acciones son deseables, ¿serán suficientes por sí mismas para generar una mayor convergencia en los niveles de desarrollo? A la luz de las consideraciones anteriores, la respuesta es posiblemente negativa. Esta agenda no se aboca a las asimetrías que tienden a generar divergencia en los patrones de desarrollo. Por otra parte, la "nivelación del campo de juego" implica restricciones a los países en desarrollo, particularmente de ingreso medio, que los propios países industrializados nunca enfrentaron en etapas anteriores de su historia: estándares de protección a la propiedad intelectual característicos de países generadores de tecnología y limitaciones a la adopción de políticas orientadas a promover nuevos sectores productivos, tanto para el mercado interno como para la exportación (Chang, 2001). Finalmente, los problemas que genera una institucionalidad financiera internacional claramente incompleta, unida a la política de intervenir menos en los mercados financieros para evitar el "riesgo moral" y a medidas orientadas a reducir el riesgo en que incurren los grandes agentes financieros (la mayor ponderación del riesgo que proponen los nuevos criterios del Comité de Basilea de regulación bancaria) pueden terminar contribuyendo a "resolver" el problema de la volatilidad de los flujos de capital por la vía más indeseable posible: elevando indebidamente el costo del endeudamien- to externo de los países en desarrollo, lo que puede conducir a una reducción permanente de los flujos de capital hacia ellos.

Así las cosas, el diseño de una agenda internacional equilibrada pasa inevitablemente por corregir las asimetrías del sistema económico internacional con acciones en tres frentes: i) aplicar mecanismos que aceleren la "propagación de progreso técnico" desde el centro: la transferencia de tecnología, a través de distintas formas de "trato especial y diferenciado"; ii) contribuir a través de las instituciones financieras internacionales a aumentar los márgenes con que cuentan los países en desarrollo para adoptar políticas macroeconómicas anticíclicas, a contrarrestar la concentración del crédito, poniendo recursos a disposición de los países y agentes que no tienen acceso al crédito en los mercados privados internacionales, y a acelerar el desarrollo financiero en los países en desarrollo, como única forma de compensar a largo plazo las asimetrías que caracterizan al sistema financiero internacional, y iii) garantizar que la movilidad internacional de mano de obra reciba la misma atención en la agenda global que la movilidad internacional de capitales.

\section{El desarrollo desde dentro}

En la visión de Prebisch, la importancia de corregir las asimetrías que caracterizan el sistema económico internacional implicaba, en cualquier caso, el reconocimiento del papel igualmente decisivo de las políticas adoptadas por los propios los países en desarrollo para transformar sus estructuras económicas y sociales. La corrección de estas asimetrías contribuía a construir un "ambiente facilitador" para los esfuerzos por generar un "desarrollo desde dentro".

A nivel institucional, hoy se reconoce ampliamente que la estrategia de desarrollo debe contribuir a generar pactos sociales sólidos, que garanticen la estabilidad política; sistemas legales no discrecionales y formas de comportamiento de los agentes que confieran seguridad a los contratos; y una burocracia estatal imparcial y relativamente eficiente. Sin embargo, aunque estos marcos institucionales sirven como telón de fondo para el proceso de desarrollo, no explican los impulsos concretos al crecimiento que experimentan las economías, ni tampoco su agotamiento. ${ }^{12}$ Estos

\footnotetext{
${ }^{12}$ En la terminología de Maddison (1991), se refieren a la "causalidad última" y no a la "causalidad inmediata" del crecimiento económico. Véase también Ocampo (2001b).
} 
impulsos están relacionados más directamente con los temas mencionados en las secciones anteriores: la reducción de las vulnerabilidades macroeconómicas y financieras, la velocidad de transformación de la estructura productiva, y las conexiones entre los patrones de crecimiento y la equidad, en especial la "heterogeneidad estructural" de los sectores productivos.

\section{a) Reducción de la vulnerabilidad macroeconómica y financiera}

Como ya se ha señalado, el énfasis en el primero de estos campos se ha venido desplazando de las perturbaciones comerciales, en las cuales concentró en gran medida su atención Prebisch, hacia aquellas de origen financiero. En esta materia, la experiencia latinoamericana de las últimas décadas permite deducir varios "hechos estilizados" que deben tenerse en cuenta en el diseño de mejores políticas macroeconómicas (CEPAL, 2000 y 2001b; Ffrench-Davis, 1999; Ocampo, 2000):

i) No existe una definición simple de estabilidad macroeconómica. El retorno a la disciplina fiscal y a tasas de inflación bajas no se ha traducido, en efecto, en mayor estabilidad en términos reales, es decir, del crecimiento del PIB y del empleo. Los avances mencionados tampoco se han reflejado en mejores resultados de las cuentas externas, debido a que los déficit privados no se han mantenido bajo control. Por lo tanto, la preocupación por la solidez de las cuentas fiscales debe estar acompañada de medidas que impidan la acumulación de déficit privados insostenibles.

ii) La inestabilidad real también es costosa. Genera estrategias defensivas por parte de las empresas, que postergan sus decisiones de invertir, originando así efectos adversos sobre el crecimiento económico. En las fases descendentes del ciclo hay, además, pérdidas irreversibles de activos empresariales, tanto tangibles como intangibles (conocimientos tecnológicos y organizacionales "tácitos", contactos comerciales, reputación de la empresa), particularmente cuando están acompañados de crisis financieras. Por estos motivos, hay una relación inversa entre crecimiento económico e inestabilidad real. Como veremos más adelante, a lo largo del ciclo se producen también importantes asimetrías sociales.

iii) En materia financiera, los balances son tan importantes como los flujos. En efecto, las crisis financieras se han originado no sólo en déficit en cuenta corriente insostenibles, sino también en estructuras financieras inadecuadas en el sector público y, particularmente, en el sector privado, es decir, excesivamen- te cargadas hacia pasivos de corto plazo o sujetas a riesgos de aumento de las tasas de interés o de los ritmos de devaluación.

iv) En ausencia de una política fiscal con un horizonte de mediano o largo plazo, apoyada por las instituciones apropiadas (fondos de estabilización), el mantenimiento de bajos déficit fiscales es estrictamente una política procíclica. Ella induce a expansiones del gasto durante los períodos de auge, basadas en ingresos públicos transitorios. Los recortes en función de las caídas coyunturales de los ingresos acentúan, con posterioridad, las crisis y, por esta vía, la reducción de los ingresos públicos.

v) En los países en desarrollo, la autonomía monetaria es limitada bajo cualquier régimen cambiario. Este hecho siempre se ha reconocido en relación con los sistemas de cambio fijo o semifijo. Sin embargo, lo mismo acontece cuando la tasa de interés se maneja en forma procíclica en regímenes de tipo de cambio variable, con el propósito de evitar las fluctuaciones del tipo de cambio. Dado el papel esencial que cumple el tipo de cambio en economías abiertas, determinante tanto de la competitividad como del nivel de precios, algún grado de intervención en los mercados cambiarios puede ser necesario, pero el manejo procíclico de la tasa de interés es ciertamente un camino inadecuado. Por este motivo, pese a sus limitaciones, la regulación prudencial de los flujos de capital puede agregar importantes grados de autonomía a la política monetaria.

vi) La credibilidad no necesariamente se construye con "pilotos automáticos". En la medida en que los flujos de capital son procíclicos, la renuncia explícita a la autonomía en materia de políticas (como la adopción de regímenes de convertibilidad o una moneda extranjera) puede traducirse en ciclos económicos más intensos. En tal caso, es posible que el mercado no valide, mediante una reducción del riesgo país, el hipotético aumento de "credibilidad". Por otra parte, se pierden grados de libertad para enfrentar choques externos o internos. En ambas circunstancias, los agentes privados pueden poner en duda la sostenibilidad de las propias reglas y los elevados costos de su ruptura. Por estos motivos, la administración prudente de la flexibilidad macroeconómica puede ser, a la larga, un mejor camino para construir una mayor credibilidad de las políticas que la adopción de reglas excesivamente rígidas.

Estas lecciones indican que el concepto de estabilidad macroeconómica debe ampliarse, para incluir no sólo la estabilidad de precios y el mantenimiento 
de déficit fiscales bajos, sino también la estabilidad de los ritmos de crecimiento económico, del empleo, de las cuentas externas y de los sistemas financieros nacionales, así como horizontes plurianuales en el diseño de la política fiscal. Implican también que es necesario reconocer que las autoridades enfrentan disyuntivas complejas para garantizar una estabilidad macroeconómica en este sentido amplio del término, ya que no existe una asociación simple entre sus distintos componentes.

La línea más promisoria sugiere que, para lograr este resultado, es necesario combinar en forma pragmática tres conjuntos de políticas. El primero de ellos está integrado por políticas macroeconómicas - fiscales, monetarias y cambiarias - consistentes y flexibles, destinadas tanto a evitar que los agentes públicos o privados acumulen deudas cuantiosas o que se produzcan desequilibrios en los precios macroeconómicos más relevantes (el tipo de cambio y las tasas de interés) y en el precio de los activos fijos y bursátiles durante los períodos de bonanza, como a facilitar la reactivación durante las crisis. El segundo es un riguroso sistema de regulación y supervisión prudencial de los sistemas financieros, con una clara orientación anticíclica, que establezca normas más estrictas en los períodos de euforia financiera para contrarrestar los riesgos cada vez mayores en que incurren los intermediarios durante estos períodos. El tercer elemento es una "política de pasivos" destinada a asegurar que se mantenga un perfil adecuado de vencimientos de las deudas interna y externa de los sectores público y privado (CEPAL, 2000, cap. 8; Ocampo, 2000).

Las regulaciones prudenciales de la cuenta de capitales, que se aplican en períodos de euforia para evitar el endeudamiento excesivo, pueden desempeñar un papel esencial como "política de pasivos", fomentando una estructura de financiamiento externo sesgada hacia pasivos de largo plazo, pero también, como ya lo hemos señalado, como un instrumento que proporciona una mayor autonomía a la política monetaria para adoptar políticas anticíclicas. A largo plazo, sin embargo, el instrumento principal para romper las asimetrías que caracterizan el sistema financiero internacional, a las cuales está asociado el comportamiento macroeconómico procíclico de los países en desarrollo, es un desarrollo financiero profundo en dichos países, que garantice un desarrollo adecuado de los segmentos de largo plazo del mercado y también de los mercados secundarios de los títulos correspondientes.

El manejo de las políticas macroeconómicas anticíclicas no es una tarea fácil, ya que los mercados financieros internacionales generan fuertes incentivos a gastar en exceso durante los períodos de euforia financiera y a ajustarse en exceso durante las crisis. Por ello, las políticas macroeconómicas anticíclicas de los países en desarrollo deben estar respaldadas por una institucionalidad internacional apropiada. En particular, las instituciones financieras internacionales deben asumir la función esencial de compensar el efecto procíclico de los mercados financieros, suavizando en su origen los ciclos financieros mediante una regulación adecuada en los países industrializados, y también ofrecer mayores grados de libertad para que los países adopten políticas anticíclicas, tanto mediante una vigilancia adecuada e incentivos que disminuyan los riesgos macroeconómicos y financieros en los períodos de euforia, como a través de mecanismos que permitan suavizar los ajustes frente a interrupciones abruptas de los flujos de capital (Eatwell y Taylor, 2000; Ocampo, 2001c).

\section{b) Una transformación productiva dinámica}

Una mayor estabilidad macroeconómica, aun en el sentido amplio en que hemos definido este término, es condición necesaria pero no suficiente para garantizar un rápido crecimiento económico. Esto es precisamente lo que señalan las diferentes variantes históricas del pensamiento económico estructuralista, de las cuales hace parte Prebisch, al destacar que distintos sectores económicos tienen una capacidad muy diferente de "propagar el progreso técnico" y, por ende, de dinamizar la economía, generando una estrecha relación entre dinámica estructural, inversión y crecimiento económico. El liderazgo que ejercen algunos sectores y empresas, que conforman los "núcleos dinámicos" en cada momento del tiempo, es, en esta visión, el elemento esencial del crecimiento económico.

Muchas escuelas de pensamiento han analizado las características de esta dinámica estructural. Algunas de ellas han señalado que, debido a la complementariedad (encadenamientos) entre empresas y sectores productivos, los efectos macroeconómicos y distributivos del crecimiento de un sector pueden provocar una brusca aceleración del proceso de crecimiento o pueden detenerlo (Rosenstein-Rodan, 1943; Taylor, 1991; Ros, 2000). Tal dinámica sectorial puede dar origen a sucesivas fases de desequilibrio, como lo señalara Hirschman (1961). La dinámica tecnológica puede generar, a su vez, procesos de "destrucción creativa", para utilizar la terminología de Schumpeter (1962, cap. VIII). Dado que el conocimiento técnico, y el conocimiento en general, no se trasmiten por medio de manuales 
detallados, la trayectoria de crecimiento de las empresas pasa por un intenso proceso de adaptación y aprendizaje, intrínsecamente vinculado a la experiencia que se adquiere en el proceso productivo y que determina en gran medida la acumulación de conocimientos técnicos, comerciales y organizacionales (Katz, 1976; Amsden, 2001).

Aunque se podrían emplear otras formulaciones, una que capta la esencia de la dinámica estructural se centra en dos conceptos fundamentales: i) las innovaciones y sus procesos asociados de aprendizaje; y ii) el desarrollo de encadenamientos productivos (CEPAL, 2000, cap. 9; Ocampo, 2001b). En este contexto, debe considerarse como "innovación" toda nueva forma de hacer las cosas: las nuevas tecnologías, pero también el desarrollo de nuevos sectores o productos, nuevas estrategias de comercialización y la apertura de nuevos mercados. En los países en desarrollo, muchas de estas "innovaciones" están asociadas a la exitosa adaptación de tecnologías y actividades desarrolladas anteriormente en el mundo industrializado, en este último caso mediante la sustitución de importaciones, la promoción de exportaciones o una combinación de ambas. Todas las innovaciones, en este sentido amplio del término, conllevan procesos de aprendizaje y difusión, muchos de los cuales se caracterizan por la generación de economías de escala dinámicas. Esto se aplica por igual a la innovación tecnológica y al desarrollo de nuevos sectores productivos y también de nuevas redes de mercado, donde dichas economías están asociadas a la reducción de los costos de transacción a lo largo del tiempo. El segundo concepto resalta el papel de las externalidades que los agentes y sectores económicos generan entre sí (Hirschman, 1961) y que determinan el grado de "competitividad sistémica" de las estructuras productivas pertinentes (CEPAL, 1990).

Estas ideas han sido utilizadas recientemente por diferentes autores para señalar la necesidad de una estrategia de desarrollo productivo como ingrediente fundamental de una economía en desarrollo dinámica, un tema antiguo de la literatura sobre "industrialización tardía”. Siguiendo una vieja tradición en política industrial, Chang (1994) ha resaltado la importancia de reducir los "costos de coordinación" que caracterizan el desarrollo de nuevos sectores sujetos a importantes complementariedades. Rodrik (1999) ha señalado la importancia de una "estrategia nacional de inversiones" que permita dar un impulso inicial al crecimiento, en tanto que la CEPAL (2000) se ha referido a la necesidad de adoptar una estrategia de transformación estructural. Amsden (2001) ha destacado, por su parte, la fun- ción crucial de una estrecha colaboración entre el Estado y el sector empresarial, y la necesidad de "mecanismos de control recíproco" que establezcan un vínculo entre incentivos y resultados, para que la primera no se traduzca simplemente en una fuente de "captación de rentas".

Esta interpretación pone de relieve una de las características más importantes de los casos de desarrollo exitoso del pasado: un fuerte proceso de industrialización basado en una estrecha y firme colaboración entre el Estado y el sector privado. ¿Será posible que la apertura de los mercados elimine la necesidad de adoptar políticas activas de desarrollo productivo?

Los resultados alcanzados hasta el momento en América Latina no son alentadores en este sentido. En efecto, el mayor dinamismo exportador de la historia económica de la región estuvo acompañado, en la última década del siglo XX, de un crecimiento económico mediocre. Los elementos "destructivos", derivados de la desintegración de los encadenamientos productivos nacionales y de los sistemas nacionales de innovación, han sido más fuertes que las oportunidades generadas por la expansión de las empresas trasnacionales o por patrones de desarrollo exportador altamente intensivos en insumos importados o en recursos naturales (CEPAL, 2001a). El gráfico 1 sintetiza esta realidad, al mostrar que el ritmo de crecimiento económico en los años noventa fue más de dos puntos inferior al de los años cincuenta a setenta, pero generó un déficit comercial similar al de los años setenta y superior al de las dos décadas anteriores. Más aún, como lo ha señalado la UNCTAD (1999, cap. IV), en el mundo en desarrollo este desplazamiento adverso de la relación entre crecimiento económico y balanza de comercio es virtualmente universal.

\section{GRAFICO 1 \\ Balanza comercial y tasa de crecimiento del PIB}

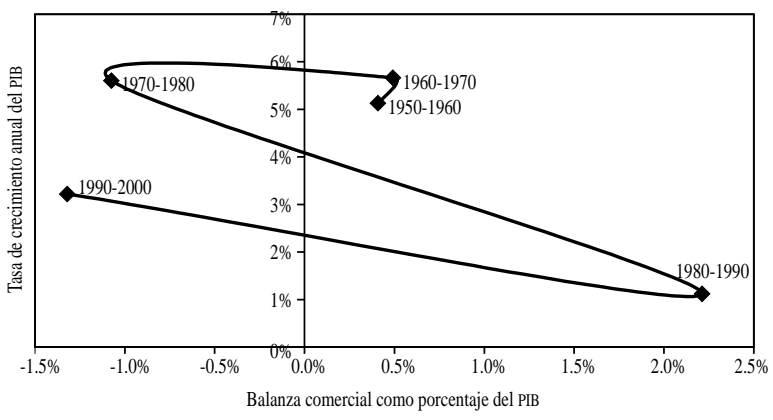

Fuente: CEPAL 
Estas consideraciones destacan la importancia de combinar la estabilidad macroeconómica, en el sentido amplio del término, con una política activa de desarrollo productivo. De acuerdo con las consideraciones anteriores, los dos elementos esenciales de la estrategia son: i) acelerar el ritmo de innovaciones, incluidos la transferencia de tecnología, el desarrollo de nuevos sectores productivos y la conquista de nuevos mercados, y apoyar los procesos de aprendizaje que caracterizan todos estos procesos; y ii) ayudar a reducir los costos de coordinación para explotar las sinergias entre empresas y sectores productivos que contribuyen a generar "competitividad sistémica". Esta política requiere un importante esfuerzo institucional y organizacional, con miras a desarrollar los instrumentos adecuados para las economías abiertas de hoy y, por ende, con un sesgo hacia la inserción activa en los mercados internacionales. Estos esfuerzos son tanto más significativos cuanto que los viejos aparatos de intervención fueron desmantelados o se debilitaron significativamente durante la fase de liberalización de las economías.

Una política de este tipo requiere también que la comunidad internacional reconozca dichas estrategias como un componente esencial de un crecimiento dinámico de los países en desarrollo. A la luz de los problemas que enfrentan actualmente dichos países para garantizar una transformación productiva dinámica, esto implica un "trato especial y diferenciado" en tres áreas: i) esquemas de protección a la propiedad intelectual que ayuden a fomentar la transferencia de tecnología; ii) fomento temporal a industrias de sustitución de importaciones; y iii) especialmente en las condiciones actuales, fomento temporal de nuevas exportaciones ("industrias incipientes de exportación”), a través del uso de incentivos orientados a diversificar la oferta exportadora, así como de mecanismos que permitan aumentar los contenidos nacionales de las exportaciones (por ejemplo, a través de acuerdos sectoriales apoyados por convenios de desempeño exportador). Todo esto exige, como es obvio, buscar los instrumentos apropiados, con el fin de evitar que estos mecanismos generen entre los países una competencia estéril por atraer industrias susceptibles de relocalización.

\section{c) Los efectos de las transformaciones económicas sobre la equidad \\ La contundencia de la observación de Prebisch} sobre las dificultades que ha enfrentado históricamente la industrialización en los países en desarrollo, y en América Latina en particular, para absorber mano de obra se resume en el gráfico 2. Tanto al comienzo (1950) como al final (1980) de su fase de industrialización más acelerada, la participación del empleo industrial en el empleo total fue inferior en América Latina en cerca de diez puntos porcentuales a lo que había sido típico en los países de la Organización de Cooperación y Desarrollo Económicos (OCDE) cuando, alrededor de medio siglo antes, habían alcanzado los mismos niveles de producto interno bruto por habitante. El excedente de mano de obra fue absorbido primero por el sector rural, pero ya para 1980 estaba mayoritariamente empleado en servicios urbanos. Dada la forma de absorción del excedente de fuerza de trabajo característica de mediados del siglo XX, Prebisch (1951) asoció dicho fenómeno con el deterioro de los precios de las materias primas. Tres décadas más tarde, éste se había convertido en América Latina en una fuente de presión sobre la distribución del ingreso a nivel urbano.

GRAFICO 2

Estructura sectorial del empleo: América Latina 1950-1980 y OCDE 1870-1950
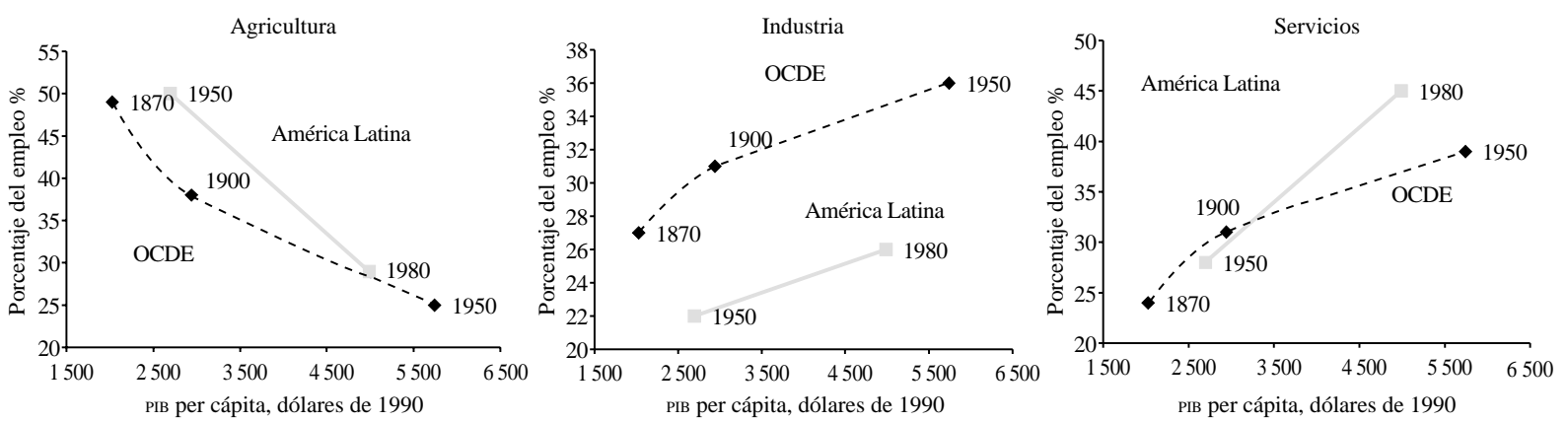

Fuente: Maddison $(1989,1995)$. 
El concepto de "heterogeneidad estructural", que desarrolló Pinto (1970) para captar este fenómeno, es ciertamente preferible al tradicional de "dualismo", porque la heterogeneidad que caracteriza a los países y las sociedades en desarrollo no puede describirse en términos del contraste entre un "sector moderno" y un "sector tradicional", y porque los sectores de baja productividad se crean y transforman constantemente y sólo unos pocos segmentos que van quedando rezagados pueden definirse como "tradicionales". Esto fue lo que sucedió en América Latina en los años noventa: la región generó más empresas de "clase mundial", capaces de integrarse exitosamente a la economía mundial, muchas de ellas filiales de empresas trasnacionales; al mismo tiempo aumentaron las actividades de baja productividad, que absorbieron a siete de cada diez trabajadores urbanos (CEPAL, 2001a y 2001d).

La heterogeneidad estructural implica la inexistencia de mecanismos automáticos que garanticen que la rápida innovación tecnológica en sectores dinámicos fomente un crecimiento económico general. En ausencia de encadenamientos internos adecuados o cuando predominan los efectos "destructivos" de la reestructuración productiva y las estrategias microeconómicas defensivas que los acompañan, la heterogeneidad estructural puede incluso acentuarse. Si esto acontece, los efectos sobre el crecimiento económico serán débiles y se experimentarán presiones adicionales sobre el empleo y la equidad (véase, por ejemplo, Taylor y Vos, 2001).

Los encadenamientos entre la modernización de los sectores dinámicos y el resto de la economía son, por lo tanto, importantes, no sólo para el crecimiento sino también para la equidad. Las estrategias de desarrollo productivo pueden desempeñar un papel trascendental en fomentar tales encadenamientos. Asimismo, la evidencia demuestra que una buena distribución de activos, que genere un universo de empresas pequeñas sólidas, está asociada con una mejor distribución del ingreso y una menor concentración del poder en general. Por lo tanto, las políticas destinadas a democratizar el acceso a los activos productivos - capital, tecnología, capacitación y tierras - de los pequeños productores rurales y urbanos tienen trascendental importancia, tanto en términos de crecimiento como de equidad.

A estos encadenamientos de carácter estructural se agregan otros asociados a la volatilidad macroeconómica: las importantes asimetrías en los efectos sociales que genera el ciclo económico. En efecto, en las fases descendentes se destruyen empleos y aumenta la incidencia de la pobreza; cuando se retorna al nivel del PIB por habitante anterior a la crisis, no necesariamente se regresa a los mismos niveles de desempleo ni de pobreza. Esto es lo que indica ciertamente la historia latinoamericana de las dos últimas décadas: pese a que el PIB por habitante retornó a mediados del decenio de 1990 a los niveles anteriores a la crisis de la deuda, todavía a fines de la década no se había llegado a los niveles de pobreza de entonces. Cabe agregar que a nivel individual, el desempleo afecta las trayectorias laborales en forma permanente: se pierde experiencia, reputación y conexiones laborales de tal forma que, cuando los trabajadores regresan al mundo laboral, obtienen remuneraciones significativamente más bajas o sólo logran reengancharse en el mercado a través de trabajos por cuenta propia (CEPAL, 2001d). Los problemas son obviamente más críticos cuando la necesidad de complementar los ingresos familiares obliga a los hijos a abandonar el estudio, con lo cual se afectan permanentemente sus propias trayectorias laborales. Por lo demás, en ausencia de instituciones que protejan a los trabajadores contra el desempleo, la inestabilidad en la demanda de mano de obra es, en sí misma, costosa en términos sociales.

Estas interconexiones de los patrones de crecimiento económico con la equidad resaltan un hecho básico: la equidad descansa, sin duda, sobre una política social muy activa, pero no únicamente sobre ella. Los ritmos y la estabilidad del crecimiento económico también importan y, muy especialmente, la forma como los patrones de transformación productiva contribuyen a reducir o a ampliar la "heterogeneidad estructural". Por este motivo, aun esfuerzos ambiciosos en materia educativa se verán frustrados si no hay una generación dinámica de empleos de calidad. En tales condiciones, las conexiones hipotéticas entre acumulación de capital humano y crecimiento no se materializan y se genera simplemente una mayor "devaluación educativa" (ocupación de los mismos puestos de trabajo por personas de mayor nivel educativo) o emigración de mano de obra educada hacia el exterior.

Por lo demás, la persistente e incluso creciente informalidad urbana y el aumento de la inestabilidad laboral hacen evidente la importancia de políticas de seguridad social universales, solidarias e integrales, que incluyan mecanismos orientados a incorporar a los trabajadores informales y, de acuerdo con el grado de desarrollo de los países, constituyan gradualmente seguros de desempleo. También es importante poner en marcha mecanismos que promuevan una mayor adaptación de la fuerza de trabajo al cambio tecnológico y 
al ciclo económico, que evite que el desempleo sea el mecanismo básico de ajuste; en particular, mecanismos que combinen políticas agresivas de capacitación laboral con el "diálogo social" a nivel de país, sector y empresa para reestructurar los sectores productivos en un marco de estabilidad del empleo (CEPAL, 2000, cap. 5). Las redes de protección social y los esquemas permanentes que permitan poner en marcha con oportunidad programas de generación de empleos de emergencia deben visualizarse, en este contexto, como instrumentos intermedios, que deben evolucionar en el largo plazo hacia sistemas integrales de seguridad social.

Las interconexiones descritas muestran, además, la importancia de diseñar marcos integrados de política. Uno de los puntos más débiles en esta esfera es la falta de instituciones que lo faciliten e incluso la tendencia que ha prevalecido en las dos últimas décadas a reforzar asimétricamente las instituciones macroeconómicas, por encima no sólo de las sociales, sino también de las responsables de las políticas de desarrollo productivo. Es necesario, por ello, reorientar radicalmente los esquemas institucionales para consolidar sistemas que permitan una coordinación entre las autoridades económicas y sociales, en los que las prioridades sociales se incorporen en el centro del diseño de la política económica, y se "visibilicen" los efectos sociales de las políticas económicas, tanto coyunturales como estructurales.

Cabe anotar que las consideraciones anteriores no agotan ciertamente los nexos positivos que puedan existir entre equidad y desarrollo, que aparte las interacciones entre capital humano y empleo de calidad, y los efectos beneficiosos de una mejor distribución de los activos productivos, pueden incluir encadenamientos favorables de economía política, efectos positivos en el mercado de capitales y nexos entre cohesión social, inversión y productividad. Las relaciones entre equidad y desarrollo constituyeron uno de los temas favoritos de la literatura sobre desarrollo de los años sesenta; afortunadamente, han vuelto a ocupar un primer plano en el debate económico reciente (véase, por ejemplo, Ros, 2000, cap. 10). Debe anotarse, sin embargo, que no todas las conexiones son positivas. De hecho, la obra tardía de Prebisch (1976, 1981 y 1987) está imbuida de consideraciones, basadas en la experiencia latinoamericana de aquellos años, sobre la forma como la presión sobre los niveles salariales y el gasto público puede generar formas de inestabilidad macroeconómica que terminan por interrumpir el crecimiento económico.

\section{La integracion regional}

Desde el punto de vista de las visiones clásicas de la integración, asociadas al pensamiento de Prebisch, la historia de la integración latinoamericana contiene una doble paradoja. Por una parte, las fuerzas proteccionistas que predominaron en la región en los años sesenta y setenta generaron procesos de integración que, en lo fundamental, restringieron su alcance a sectores no competitivos. De esta manera, una de las virtudes fundamentales de la integración, la racionalización de la sustitución de importaciones (CEPAL, 1959), sólo se alcanzó en forma muy limitada. Además, durante la crisis de la deuda de los años ochenta, los esquemas de integración regional estuvieron a punto de sucumbir ante el uso generalizado de la protección y de las devaluaciones competitivas como instrumentos de ajuste de las economías.

El vigor que caracterizó el proceso de integración regional desde fines de los años ochenta fue igualmente paradójico. En efecto, en las visiones más ortodoxas que dominaban entonces las concepciones del desarrollo, la integración era vista como una fuente de distorsión en los flujos de comercio. La coincidencia de los procesos de apertura comercial unilateral con la revitalización de la integración regional fue, por lo tanto, una concesión histórica a las visiones que habían servido para justificar la integración regional en épocas anteriores y, en particular, a la idea de que la integración era una fuente de creación más que de desviación de comercio y, más aún, una fuente de flujos de comercio con mayores contenidos tecnológicos que los que resultan de la apertura unilateral.

Estas virtudes se han materializado en la práctica. En efecto, el crecimiento del comercio intrarregional fue extremadamente dinámico entre 1990 y 1997, en particular en los dos acuerdos de integración sudamericanos, el Mercosur y la Comunidad Andina, donde se multiplicó por 5 y por 4.2 veces, respectivamente, durante estos años. Como resultado de ello, además, se revirtió la relación histórica según la cual la intensidad del comercio intrarregional era mayor para las economías más pequeñas. Hacia 1997, los mayores flujos relativos de comercio intrarregional se concentraban, de hecho, en el Mercosur. En todos los acuerdos regionales de integración existe, sin embargo, un largo camino por recorrer si el punto de referencia es la Unión Europea, donde el comercio intrarregional ha representado en torno al $60 \%$ del comercio total durante las últimas décadas. Cabe anotar, por otra parte, que el comercio intrarregional ex- 
perimentó una caída pronunciada con la crisis asiática. Los más afectados fueron los flujos entre países sudamericanos, que se recuperaron, sin embargo, en forma vigorosa en el 2000. Esto implica, por lo tanto, que las elasticidades de dicho comercio a la actividad económica son hoy en día muy elevadas (CEPAL, 2001c).

Por otra parte, los flujos comerciales intrarregionales se concentran en bienes industriales, especialmente aquellos con mayor contenido tecnológico. Este patrón es particularmente evidente cuando se excluye México, cuyas ventas de productos manufacturados a Estados Unidos han crecido rápidamente a partir de la suscripción del Tratado de Libre Comercio de América del Norte. En 1999, por ejemplo, el $81 \%$ de las exportaciones intrarregionales correspondía a productos manufactureros, en comparación con el $65 \%$ en el conjunto de las ventas externas (excluyendo a México). El contraste es mayor cuando sólo se incluyen productos no tradicionales. Como resultado, el comercio intrarregional absorbía entonces el 35\% de las exportaciones manufactureras, concentrado en los productos de mayor contenido tecnológico.

La visión según la cual existe una complementariedad entre los procesos generales de apertura comercial y la integración fue bautizada por la CEPAL (1994) con el nombre de "regionalismo abierto". Esta complementariedad indica que, pese a los avances en materia de información y comunicaciones, las economías externas y de escala (incluidas las economías de especialización) y los costos de transacción asociados a la distancia siguen siendo importantes. Estos últimos incluyen no sólo los costos de transporte, sino también aquellos asociados a la creación de redes comerciales, en especial las que involucran a empresas pequeñas y medianas. Además, los procesos regionales permiten profundizar la armonización de las normas que afectan la actividad económica y promueven relaciones económicas más profundas que también tienen efectos sobre el comercio. Por este motivo, las redes regionales crean comercio en forma adicional al que se logra con la apertura comercial unilateral.

Cabe anotar, sin embargo, que el nuevo regionalismo difiere de sus homólogos del pasado por el aumento en el número de ámbitos involucrados y el alcance de la liberalización arancelaria. Sin embargo, la convergencia de los acuerdos existentes para conformar áreas de mayor tamaño relativo, así como la consolidación de uniones aduaneras, siguen siendo tareas pendientes. La institucionalidad existente continúa siendo, además, frágil.
Más allá del comercio, es evidente que el futuro de la integración regional depende de la "profundidad" que alcancen los acuerdos correspondientes. En efecto, el espacio para instancias regionales, en la era de la globalización, depende de dos fuerzas diferentes. La primera de ellas es el debilitamiento de la autonomía nacional, que implica que la eficacia de las políticas se traslada a instancias regionales o globales. La segunda es el peso relativo que tienen, dentro de este proceso, dos factores: i) el alcance regional -en oposición al global — de los "bienes públicos" que se busca promover; si su alcance no es estrictamente global, el principio de subsidiariedad indica que deben ser asumidos por instancias intermedias, de carácter regional; ii) la capacidad de las distintas instituciones de generar "sentido de pertenencia" (ownership); desde este punto de vista, existe una nítida ventaja en favor de las instancias subregionales y regionales.

En materia macroeconómica y financiera, queda claro que la pérdida de autonomía es creciente, requiriendo, según hemos señalado, espacios para acciones de carácter global, pero también para instancias regionales. La posible coordinación de las políticas macroeconómicas constituye una primera área posible de acción. Más aún, la demanda por acciones de este tipo se ha visto acrecentada por la gran vulnerabilidad del comercio intrarregional a los ciclos económicos recientes. No obstante, las dificultades que involucra este proceso quedaron claramente reveladas en tres décadas de experiencias acumuladas por los países europeos. Por este motivo, es evidente que los objetivos deben ser modestos en el corto plazo.

Una medida altamente complementaria con las anteriores sería el establecimiento de mecanismos para coordinar las políticas de regulación y supervisión prudencial de los sistemas financieros. Lo deseable en esta materia sería el desarrollo de mecanismos de vigilancia mutua de dichas políticas y, eventualmente, el diseño de estándares mínimos más específicos que los de Basilea. El hecho de que algunos de los principales bancos internacionales operen en varios países de la región resalta aún más la importancia de la coordinación en esta área, para brindar una regulación y supervisión más adecuada y evitar el arbitraje por parte de estos bancos entre distintos sistemas regulatorios.

Como lo ha resaltado la CEPAL (2001b), igual atención debe prestarse al desarrollo de instituciones financieras regionales y subregionales. En esta materia, América Latina y el Caribe tienen ya activos importantes en términos de una red de bancos multilaterales de desarrollo, constituida por el Banco Interamericano de 
Desarrollo, la Corporación Andina de Fomento, el Banco Centroamericano de Integración Económica y el Banco de Desarrollo del Caribe. Por otra parte, la experiencia ya acumulada por el Fondo Latinoamericano de Reservas (FLAR, antiguamente Fondo Andino de Reservas) permite pensar en la posibilidad de apoyar con recursos excepcionales, de origen regional, a los países en crisis, ya sea mediante una expansión significativa de los miembros y recursos del FLAR, o mediante acuerdos de apoyo mutuo (mediante swaps) de los bancos centrales.

El avance mismo del comercio crea, además, la demanda de armonización de los distintos esquemas regulatorios. Entre ellos se cuentan la armonización de normas técnicas, incluyendo las fitosanitarias, los códigos aduaneros, las normas de compras gubernamentales y las que regulan la prestación de servicios. En algunos de estos campos ya hay algún avance en la región. Es importante progresar complementariamente en otros, especialmente en materia de normas de competencia y de regulación de los servicios públicos. Sobre las primeras cabe mencionar que hay razones para pensar, con base en la experiencia europea, que a medida que se consoliden mercados comunes y un proceso activo de inversión intrarregional, los acuerdos regionales en materia de competencia y, eventualmente, la adopción de una política de competencia común, tienen ventajas evidentes sobre las normas de competencia desleal que regulan exclusivamente el comercio exterior. Un marco de esta naturaleza permitirá, entre otras cosas, manejar más eficazmente las eventuales prácticas anticompetitivas de grandes empresas trasnacionales.

Por otra parte, el elevado peso de los productos con mayor contenido tecnológico en el comercio intrarregional llama la atención sobre la posibilidad de acciones conjuntas para desarrollar los sectores correspondientes, obviamente sin las rigideces de los viejos (y, en gran medida, fallidos) acuerdos de complementación sectorial. Más aún, ellos deben hacer parte de esquemas más amplios de complementación de los esfuerzos de investigación y desarrollo tecnológico, que sirvan igualmente como mecanismo de transferencia de tecnología hacia los países de menor desarrollo relativo.

En el área de la infraestructura física, además del desarrollo de normativas armonizadas en transporte, energía y telecomunicaciones, resulta trascendental el desarrollo de redes de infraestructura concebidas en función de la integración regional, y no exclusivamente de la integración nacional. El desarrollo de la infraes- tructura con esta visión llama, además, la atención sobre la necesidad de visualizar los espacios como comunes, más que como nacionales. Un caso interesante en tal sentido fue la constitución, en 1994, de la Asociación de Estados del Caribe, para promover una mayor cooperación en dicha cuenca. En el caso del desarrollo sostenible se han venido dando igualmente pasos para una visión de este tipo, en este caso de ecosistemas comunes (el amazónico, el andino, el corredor mesoamericano) o de cuencas hidrográficas compartidas. También el surgimiento de distintos planes de desarrollo fronterizo es otro ejemplo de este cambio en la visión del espacio que genera la integración.

Más allá de ello, existen, además, las inmensas posibilidades que abre la integración en el campo social y político. Los acuerdos en materia cultural y educativa, algunos de ellos asociados a la primera etapa de la integración, son apenas el primer e incipiente paso hacia una agenda de integración que tenga en su centro el desarrollo social. A su vez, las "cláusulas democráticas" de los acuerdos de integración y los diversos foros que sirven hoy de marco al encuentro de los jefes de Estado de la región, así como los incipientes parlamentos subregionales y latinoamericano, son semillas de la amplia agenda de integración política.

Estas consideraciones demuestran que la integración regional, sustentada en los principios del regionalismo abierto, tiene un futuro promisorio, cuya materialización dependerá de la voluntad política de las partes. Más aún, su capacidad de sobrevivir en el contexto de un área de libre comercio hemisférica o del proceso de globalización dependerá de su capacidad para profundizarse, desarrollando la agenda que hemos delineado.

En cualquier caso, la categórica admonición de Prebisch sigue sonando con fuerza, y no únicamente en relación con el comercio sino con el conjunto del sistema económico (y político) internacional. "Hay un aspecto de importancia fundamental al que no han prestado atención suficiente los gobiernos de los países en desarrollo. Aún no hemos podido romper el aislamiento que heredaron estos países del antiguo patrón de la división internacional del trabajo. En efecto, la mayor parte del comercio mundial se ha realizado entre los propios centros. El comercio de los países en desarrollo ha convergido en los centros, pasando por alto la potencialidad enorme del comercio recíproco. Por cierto, debo recordar que desde los primeros días de la CEPAL he predicado vigorosamente la necesidad de esta reforma estructural del comercio mundial” (Prebisch, 1987, p. 29). 
Bibliografía

Aceña, M. P. y J. Reis (eds.) (2000): Monetary Standards in the Periphery: Paper, Silver and Gold, 1854-1933, Nueva York, St. Martin's Press.

Amsden, A. (2001): The Rise of "The Rest": Challenges to the West from Late Industrializing Countries, Nueva York, Oxford University Press.

Bielschowsky, R. (1998): Cincuenta años de pensamiento de la CEPAL: una reseña, CEPAL (Comisión Económica para América Latina y el Caribe), Cincuenta años de pensamiento en la CEPAL: textos seleccionados, México, D.F., Fondo de Cultura Económica.

Cárdenas, E., J. A. Ocampo y R. Thorp (eds.) (2000a): The Export Age: The Latin American Economies in the Late Nineteenth and Early Twentieth Centuries. An Economic History of Twentieth Century Latin America, vol. I, Nueva York, St. Martin's Press.

(2000b): Industrialisation and the State in Latin America: The Post War Years. An Economic History of Twentieth Century Latin America, vol. III, Nueva York, St. Martin's Press.

CEPAL (1959): El mercado común latinoamericano, E/CN.12/531, México, D.F., julio.

(1990): Transformación productiva con equidad, LC/G.1601-P, Santiago de Chile. Publicación de la Naciones Unidas, $\mathrm{N}^{\circ}$ de venta S.90.II.G.6.

(1994): El regionalismo abierto en América Latina y el Caribe. La integración económica al servicio de la transformación productiva con equidad, LC/G.1801/Rev.1-P, Santiago de Chile. Publicación de las Naciones Unidas, $\mathrm{N}^{\circ}$ de venta S.94.II.G.3.

(1998): Cincuenta años del Estudio económico, Estudio económico de América Latina y el Caribe, 1997-1998, LC/G.2032-P, Santiago de Chile.

(2000): Equidad, desarrollo y ciudadanía, LC/G.2071 (SES.28/3), Santiago de Chile.

(2001a): Una década de luces y sombras. América Latina y el Caribe en los años noventa, Bogotá, Alfaomega.

(2001b): Crecer con estabilidad: el financiamiento del desarrollo en el nuevo contexto internacional, Bogotá, Alfaomega.

(2001c): Panorama de la inserción internacional de América Latina y el Caribe, 1999-2000, LC/G.2085-P, Santiago de Chile.

(2001d): Panorama social de América Latina, 2000-2001, Santiago de Chile.

Chang, H. (1994): The Political Economy of Industrial Policy, Londres, Macmillan.

(2001): Infant industry promotion in historical perspective. A rope to hang oneself or a ladder to climb with?, trabajo presentado en el seminario La teoría del desarrollo en los albores del siglo XXI, Santiago de Chile, CEPAL, 28-29 de agosto.

Council on Foreign Relations (1999): Safeguarding Prosperity in a Global Financial System: The Future International Financial Architecture, C. A. Hills, P. G. Peterson (Chairs) y M. Goldstein (Project Director), Task Force Report, Washington, D. C., Institute for International Economics (IIE).

Eatwell, J. y L. Taylor (2000): Global Finance at Risk: The Case for International Regulation, Nueva York, The New Press.

Ffrench-Davis, R. (1999): Macroeconomía, comercio y finanzas para reformar las reformas en América Latina, Santiago de Chile, Mc.Graw Hill-Interamericana.

Furtado, C. (1961): Desenvolvimento e subdesenvolvimento, Rio de Janeiro, Editora Fundo de Cultura.

Hirschman, A. O. (1961): La estrategia del desarrollo económico, México, D.F., Fondo de Cultura Económica.
Katz, J. (1976): Importación de tecnología, aprendizaje e industrialización dependiente, México, D.F., Fondo de Cultura Económica.

(2000): Reformas estructurales, productividad y conducta tecnológica en América Latina, Santiago de Chile, CEPAL/ Fondo de Cultura Económica.

Kaul, I., I. Grunberg y M. A. Stern (comps.) (1999): Global Public Goods: International Cooperation in the 21st Century, Nueva York, Programa de las Naciones Unidas para el Desarrollo (PNUD).

Krugman, P. (1990): Rethinking International Trade, Cambridge, Massachusetts, The MIT Press.

(1999): Depression economics, Nueva York, Council on returns, Foreign Affairs, Nueva York, Council on Foreign Relations, enero-febrero.

Maddison, A. (1989): The World Economy in the 20th Century, París, Organización de Cooperación y Desarrollo Económicos (OCDE), Centro de Desarrollo.

(1991): Dynamic Forces in Capitalist Development: A Long-Run Comparative View, Nueva York, Oxford University Press.

(1995): Monitoring the World Economy 1820-1992, París, OCDE, Centro de Desarrollo.

Ocampo, J. A. (1991): Los términos de intercambio y las relaciones centro-periferia, O. Sunkel (comp.), El desarrollo desde dentro: un enfoque neoestructuralista para la América Latina, El trimestre económico, lecturas, $\mathrm{N}^{\circ} 71$, México, D.F., Fondo de Cultura Económica.

(2000): Developing Countries' Anti-Cyclical Policies in a Globalized World, Serie temas de coyuntura, $\mathrm{N}^{\circ} 13$, Santiago de Chile, CEPAL.

(2001a): Retomar la agenda del desarrollo, Revista de la CEPAL, N ${ }^{\mathrm{o}} 74$, LC/G.2135-P, Santiago de Chile, CEPAL.

(2001b): Structural Dynamics and Economic Development, Kurt Martin lecture, La Haya, Países Bajos, Instituto de Estudios Sociales, abril.

(2001c): International Asymmetries and the Design of the International Financial System, Serie temas de coyuntura, $\mathrm{N}^{\mathrm{o}}$ 15, Santiago de Chile, CEPAL.

Palma, G. (2001): Three-and-a-half cycles of 'mania, panic and [asymmetric] crash': East Asia and Latin America compared, H. Chang, G. Palma y D. H. Whittaker (eds.), Financial Liberalization and the Asian Crisis, Nueva York, Palgrave Publishers.

Pérez, C. (2001): Cambio tecnológico y oportunidades de desarrollo como blanco móvil, trabajo presentado al seminario La teoría del desarrollo en los albores del siglo XXI, Revista de la CEPAL, $N^{\circ} 75$, LC/G. 2150-P, Santiago de Chile, CEPAL, diciembre.

Pinto, A. (1970): Naturaleza e implicaciones de la 'heterogeneidad estructural' de la América Latina, El trimestre económico, vol. XXXVII(1), N 145, México, D.F., Fondo de Cultura Económica. Reproducido en CEPAL, Cincuenta años de pensamiento en la CEPAL: textos seleccionados, vol. 1, México, D.F., Fondo de Cultura Económica. 1998.

Prebisch, R. (1949): Introducción: el desarrollo económico de la América Latina y algunos de sus principales problemas, Estudio económico de América Latina, 1948, Santiago de Chile, CEPAL. Reproducido en CEPAL, Cincuenta años de pensamiento en la CEPAL: textos seleccionados, vol.1, México, D.F., Fondo de Cultura Económica. 1998.

(1951): Crecimiento, desequilibrio y disparidades: interpretación del proceso de desarrollo, Estudio económico de América Latina 1949, Santiago de Chile, CEPAL. También publicado como: "Interpretación del proceso de desarrollo 
latinoamericano en 1949", CEPAL, XXV años de la CEPAL, Santiago de Chile, 1973.

(1952): Problemas teóricos y prácticos del crecimiento económico, México, D.F., CEPAL. Reproducido en CEPAL, $X X V$ años de la CEPAL, Santiago de Chile, 1973.

(1963): Hacia una dinámica del desarrollo latinoamericano, México, D.F., Fondo de Cultura Económica.

(1964): Nueva política comercial para el desarrollo, México, D.F., Fondo de Cultura Económica.

(1976): Crítica al capitalismo periférico, Revista de la CEPAL, $\mathrm{N}^{\circ}$ 1, S76.II.G.2, Santiago de Chile, CEPAL, primer semestre.

(1981): Capitalismo periférico. Crisis y transformación, México, D.F., Fondo de Cultura Económica.

(1987): Cinco etapas de mi pensamiento sobre el desarrollo, CEPAL, Raúl Prebisch: un aporte al estudio de su pensamiento, LC/G.1461, Santiago de Chile.

Rodríguez, O. (2001): Fundamentos del estructuralismo latinoamericano, Comercio exterior, vol. 51, N² , México, D.F., Banco Nacional de Comercio Exterior (BANCOMEXT).

Rodrik, D. (1997): Has Globalization Gone Too Far?, Washington, D.C., Institute for International Economics.

(1999): The New Global Economy and Developing Countries: Making Openness Work, Policy essay, $\mathrm{N}^{\circ} 24$, Washington, D.C., Consejo de Desarrollo de Ultramar.

Ros, J. (2000): Development Theory and The Economics of Growth, Ann Arbor, Michigan, The University of Michigan Press, septiembre.
Rosenthal, G. (2001): The contribution of the Economic Commission for Latin America and the Caribbean to the United Nations intellectual legacy, Naciones Unidas, United Nations Intellectual History Project. Views from the Regional Commission: ECLAC, vol. XII, Nueva York.

Rosenstein-Rodan, P.N. (1943): Problems of industrialization of Eastern and South-Eastern Europe, The Economic Journal, vol. 53, Londres, Macmillan and Co., Ltd., junio-septiembre.

Schumpeter, J. (1962): Capitalism, Socialism and Democracy, Nueva York, Harper Torchbooks.

Studart, R. (1996): The efficiency of the financial system, liberalization and economic development, Journal of Post Keynesian Economics, vol. 18, № 2, Arkmon, Nueva York, M. E. Sharpe, Inc.

Sunkel, O. (comp.) (1991): El desarrollo desde dentro: un enfoque neoestructuralista para la América Latina, El trimestre económico, lecturas, № 71, México, D.F., Fondo de Cultura Económica.

Taylor, L. (1991): Income Distribution, Inflation and Growth, Cambridge, Massachusetts, The MIT Press.

Taylor, L. y R. Vos (2001): Liberalización de la balanza de pagos en América Latina. Efectos sobre el crecimiento, la distribución y la pobreza, en E. Ganuza, R. Paes de Barros, L. Taylor y R. Vos (eds.), Liberalización, desigualdad y pobreza: América Latina y el Caribe en los 90, Buenos Aires, Universidad de Buenos Aires/PNUD/CEPAL.

Triffin, R. (1968): Our International Monetary System: Yesterday, Today, and Tomorrow, New York, Random House.

UNCTAD (Conferencia de las Naciones Unidas sobre Comercio y Desarrollo) (1999): Informe sobre el comercio y el desarrollo, 1999, Ginebra. 